
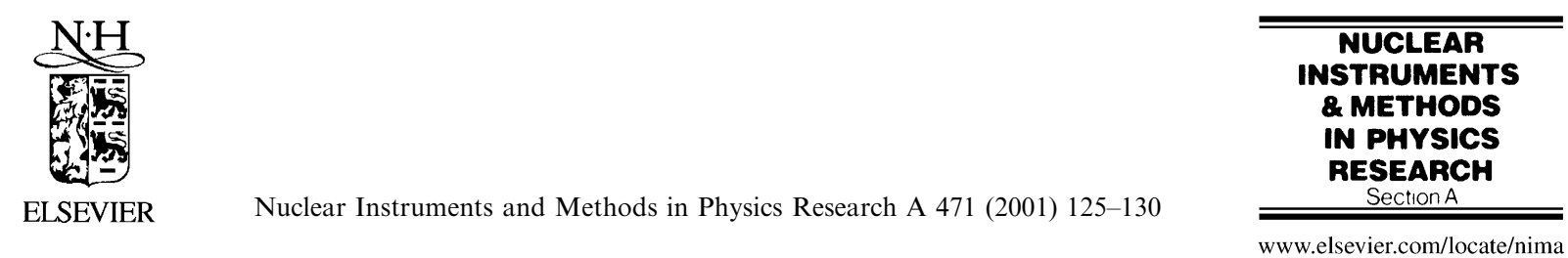

\title{
Optical readout of GEMs
}

\author{
F.A.F. Fraga*, L.M.S. Margato, S.T.G. Fetal, M.M.F.R. Fraga, \\ R. Ferreira Marques, A.J.P.L. Policarpo \\ Departamento de Física, LIP-Coimbra, Universidade de Coimbra, 3000 Coimbra, Portugal
}

\begin{abstract}
We present an overview of results from our recent studies on the use of the visible and NIR scintillation emitted by the gas electron multiplier (GEM) and on the possibility of using detectors operated with cascaded GEMs to build tracking chambers. (C) 2001 Elsevier Science B.V. All rights reserved.
\end{abstract}

\section{Introduction and motivation}

Most of the published research done in the use of gaseous scintillators was carried on in the VUV and UV zone of the spectrum, using photon counting techniques and high efficiency mixtures, and many detectors were developed. However many imaging applications can be found for integrating devices, that operate by accumulation of the level of irradiation for a frame period with a sequent dead time for read-out. The image plates and CCDs are examples of this type of detector. This last device has been perfected during the last decade, and current high performance CCD digital cameras show high sensitivity and low noise for a reasonable price without need of special fluid cooling systems.

Although the more recent developments in CCD technology have achieved high quantum efficiency down to $120 \mathrm{~nm}$ using back illuminated sensors, these devices are still expensive and of critical use.

*Corresponding author. Tel.: + 351-39-833-465; fax: + 35139-822358.

E-mail address: francisco@lipc.fis.uc.pt (F.A.F. Fraga).
Systems using this zone of the spectrum are awkward to design and operate, needing special optics and windows to couple the scintillation light to the CCD with acceptable losses.

Considering that common glass optics have good transmission in the range of standard CCDs and that electron avalanches in appropriate gas mixtures can result in the emission of light in the visible and NIR band covered by this devices, our group has been investigating the emissions of visible and NIR light by microstructures.

In the visible region we have been considering the study of scintillation emitted by microstructure detectors, but only during the last three years we have been developing a large activity on the subject. The purpose of this paper is to report our studies in the application of CCDs and conditions of operation of gaseous mixtures for efficient light emission between 400 and $1000 \mathrm{~nm}$.

\section{Previous work}

A particularly high emission of light was reported by Oed and Geltembort in 1991 [1]. The 
authors consider that the entirely different behavior of microstrips compared to wire detectors is to be sought in the different geometry of the electric field, and that operation of the devices with pure gas mixtures is possible. They also report that using a mixture of $\frac{1}{3}$ each of $\mathrm{Ar}, \mathrm{Kr}$ and $\mathrm{Xe}$ at $1050 \mathrm{mbar}$ and a gas gain of 500 , the avalanches induced by alpha particles with an energy loss of $3 \mathrm{MeV}$ in the gas produce about $8 \times 10^{5}$ photons. This localized light could be easily detected by means of a Peltier cooled CCD camera.

Our first studies were motivated by the expected application of microstructures such as microstrip, microgap, GEMs, etc., for low-cost, high resolution intermediate tracking of charged particles for the new high luminosity colliders. We considered the possibility of performing non-destructive testing of microstructure gaseous detectors using both the optical emission associated with the pure secondary scintillation, i.e., without charge multiplication, and/or scintillation accompanying the development of the electronic avalanches. The advantage of this methods is that local field configuration is looked for, a clear advantage over the methods that have been used.

The chosen mixture for thesis experiments was $\mathrm{Ar}-5 \% \mathrm{CO}_{2}$. Although the addition of $\mathrm{CO}_{2}$ decreases the light emission, the amount of emitted light in Ar has a strong dependence on the purity of the gas and a few percent of quencher stabilizes its operation [2]. The emission spectra of $\mathrm{Ar}-\mathrm{CO}_{2}$, obtained in a sphere detector operated in proportional mode, is show in Fig. 1 [3].

Our first attempts were made with MSGCs pursuing the development of a method of quality control of microstructures. Under uniform irradiation of the microstructure the light of the avalanches as seen by a CCD camera map the electric field of the device and the defects of the electrodes or substract could be easily detected. GEM tests were envisaged, as the device became available. Our first results with the GEM have also shown that the light is mainly emitted in the neighborhood of GEM channels and, as the GEM is an optically transparent device, the light emitted can be read from the side opposing the incoming electrons, without any interference with the radia-

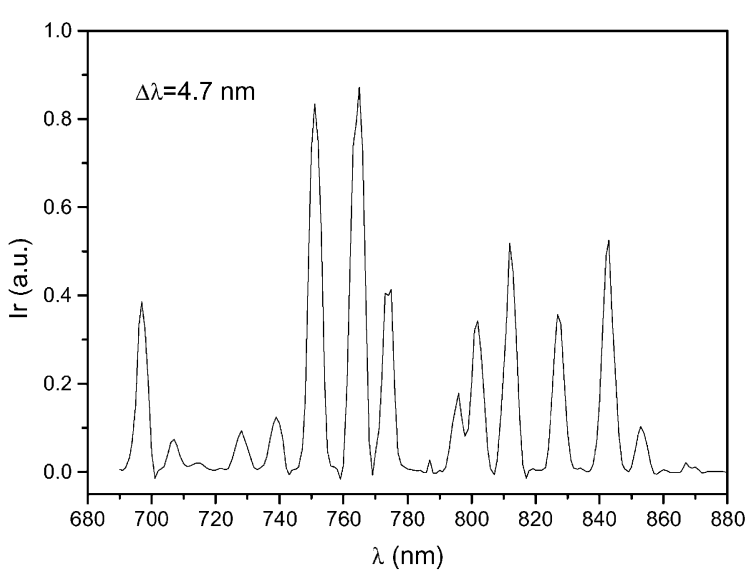

Fig. 1. NIR emission spectrum of $\mathrm{Ar}-3 \% \mathrm{CO}_{2}$ measured, under X-ray excitation, with slit widths of $1 \mathrm{~mm}(P=750$ Torr).

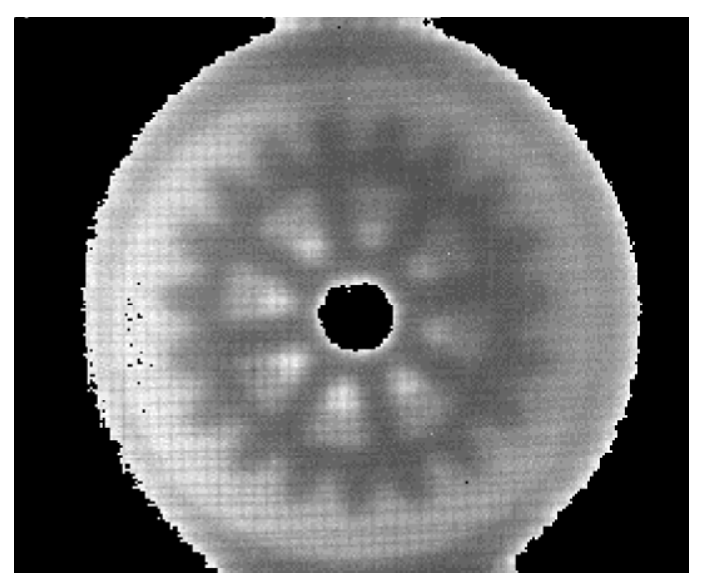

Fig. 2. Radiography of a plastic double gear, obtained with an $\mathrm{Ar}-5 \% \mathrm{CO}_{2}$ mixture; gear diameter $=25.6 \mathrm{~mm}$, thickness 4 and $8.9 \mathrm{~mm}$.

tion beam. We have now built several imaging detectors using these principles, using one or two cascaded GEM foils and a Peltier cooled CCD system and the results were presented in several conferences this year [4,2]. Fig. 2 shows a picture of several plastic gears. Position resolution is limited by the GEM granularity and the properties of the gas mixture we measured $100 \mu \mathrm{m}$ RMS using $\mathrm{Xe}-5 \% \mathrm{CO}_{2}$. Also, using the cascaded GEMs, images of acceptable quality could be obtained using moderate X-ray fluxes with exposition time as short as $1 \mathrm{~s}$. 


\section{New results on quality control of GEMs}

The uniformity of the gain of the whole foil is one important aspect of GEM performance. The GEM gain depends on the diameter of the GEM copper and kapton holes, kapton thickness and alignment of the holes. The diameter of the holes is established by the mask design, but can be affected during manufacture by local changes in the etching process. We carried on several simulations on the dependence of the gain on the variations of the GEM geometry and found that the alignment of the masks is of major importance to keep constant gain over the surface. Variations as small as $3 \mu \mathrm{m}$ can lead to changes in gain over $10 \%$, and cannot be easily checked by optical means. The measurement of the scintillation of the GEMs under uniform irradiation can be used for checking the homogeneity of gain of the whole surface with high spatial resolution. Details of a similar experimental arrangement and test procedure have been reported elsewhere [2]; we will describe it briefly, enhancing the differences from the previous setup.

A cross-section of the chamber is shown in Fig. 3. No collecting electrode was used on these experiments, the avalanche electrons were collected by the GEM outer electrode. The entrance window was made from $12 \mathrm{~mm}$ aluminized mylar foil and a $10 \times 10 \mathrm{~cm}^{2}$ GEM foil was used for the measurements. The GEM and the drift grids were supported by fiber glass frames. Considering that a maximal error of flatness of the GEM of $0.25 \mathrm{~mm}$ is possible, the absorption length was chosen to be rather large, and the drift plane was placed $25 \mathrm{~mm}$ before the GEM, in order to minimize the contributions to the local variation of primary current, making this error less than $1 \%$. The light window was a $0.5 \mathrm{~mm}$ mylar foil covering all the surface of the GEM. Both windows were checked for uniformity of transmission before being assembled. All these structures were clamped by two external aluminium frames.

The GEM, supplied by the CERN GDD Group, was manufactured from a $50 \mu \mathrm{m}$ thickness copper coated kapton foil by a chemical etching process that produces holes with a double conical shape (standard shape) [5]. The diameters of metal

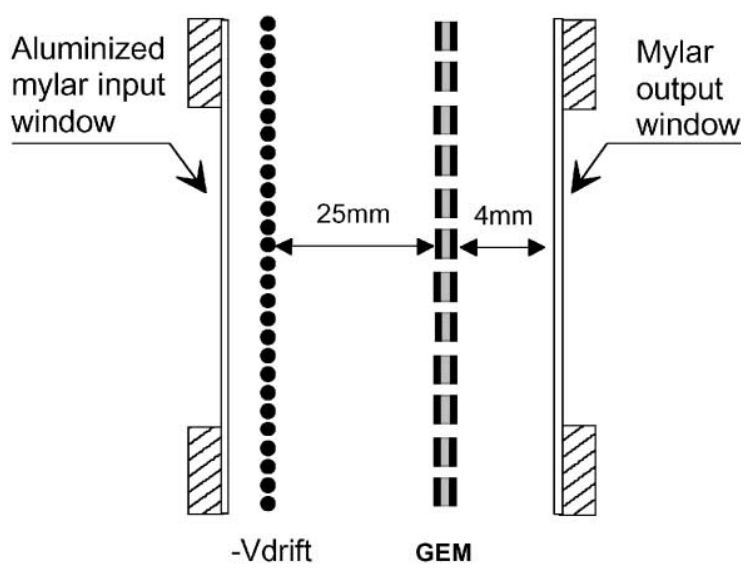

Fig. 3. The cross-section view of the detector chamber.

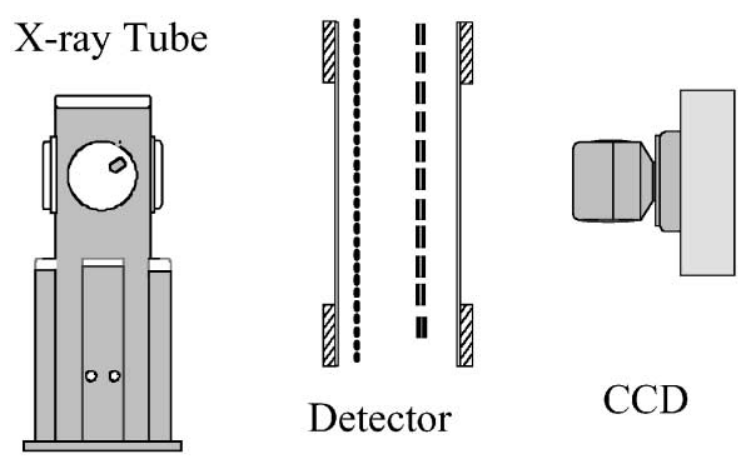

Fig. 4. Schematic diagram of the experimental arrangement.

and kapton holes were 80 and $50 \mu \mathrm{m}$, respectively. The pitch was $140 \mu \mathrm{m}$ and the optical transparency was $\approx 12 \%$. The GEM front side was grounded and the back one was operated at negative voltage of $340 \mathrm{~V}$ (gain $\approx 90$ ).

A Quantix 1400 camera, manufactured by Photometrics Ltd., was used to read the light emitted from the GEM. It uses a Peltier cooled, low noise CCD, KAF 1400 from Kodak, with $1317 \times 1035$ pixels of $6.8 \times 6.8 \mu \mathrm{m}^{2}$ and the spectral response goes from 400 to $1000 \mathrm{~nm}$. All the pictures presented in this work were taken with the CCD cooled to $-30^{\circ} \mathrm{C}$, without any fluid cooling.

A diagram of the complete system is shown in Fig. 4. The primary electrons produced by the $\mathrm{X}$-ray photons in the conversion region drift towards the GEM holes. The grid currents were 
monitored with the high voltage power supply ammeters with a resolution of $\mathrm{nA}$ and the outer GEM grid was connected to the ground through a Keithley picoammeter, allowing for precision measurement of the collected electrons current. All the electrons produced in the multiplication zone were collected at the outer electrode of the GEM, and the ratio between primary current and this current was used to calculate the GEM gain.

The chamber was irradiated by an X-ray generator with a molybdenum tube. As this tube was operated at low voltages, typically around $20 \mathrm{kV}$, the main bremsstrahlung spectrum was peaking around $13 \mathrm{keV}$. The $\mathrm{X}$-ray tube and the CCD were placed at the distances 110 and $77 \mathrm{~cm}$, respectively, from the GEM chamber to minimize the contributions due to solid angle effects in irradiation and light collection.

The CCD image in Fig. 5 shows the scintillation of the full area of a GEM foil taken with GEM voltage of $340 \mathrm{~V}$, gain 88 . The detector was uniformly irradiated with X-rays. Fig. 6 shows the distribution of collected light on the pixels of the CCD.

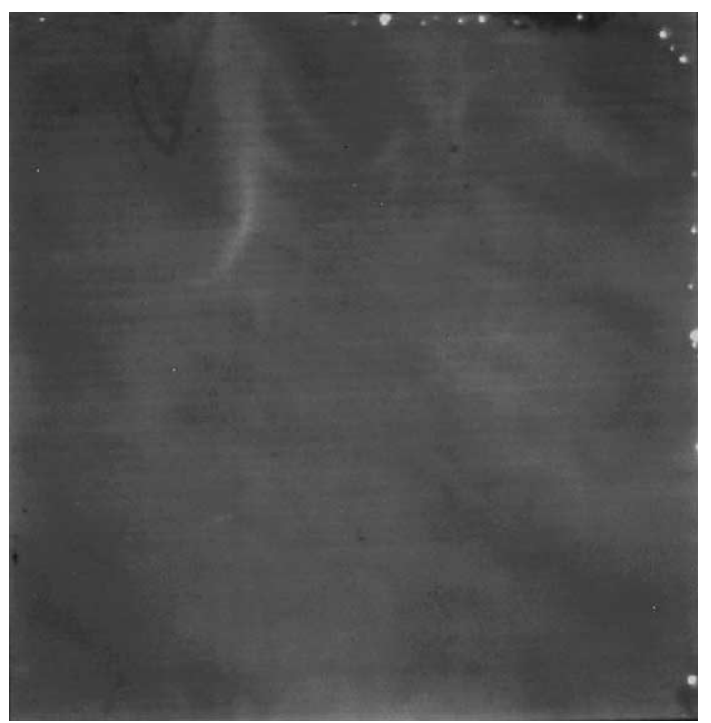

Fig. 5. Scintillation image of a $10 \times 10 \mathrm{~cm}^{2}$ GEM. The scintillation intensity maps the gain of the surface, but it is modulated by factors affecting the reflectivity of the GEM: the upper left brighter zone is due to a small wrinkle on the GEM.

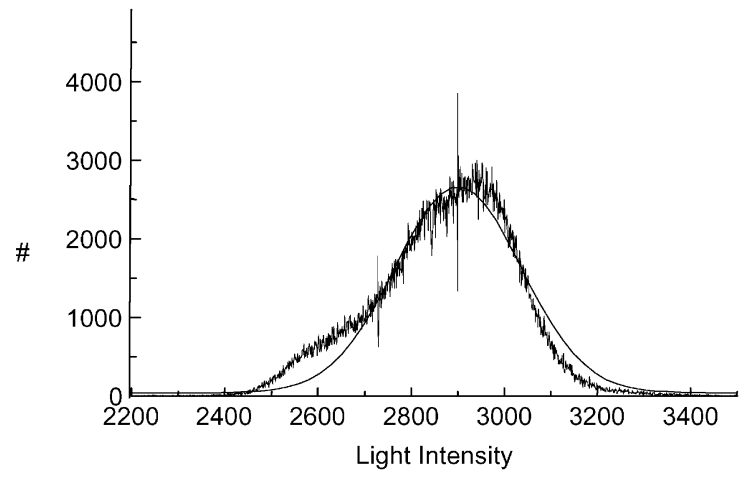

Fig. 6. Distribution of the light collected by the CCD pixels (see Fig. 5). The distribution has a RMS width of $4 \%$.

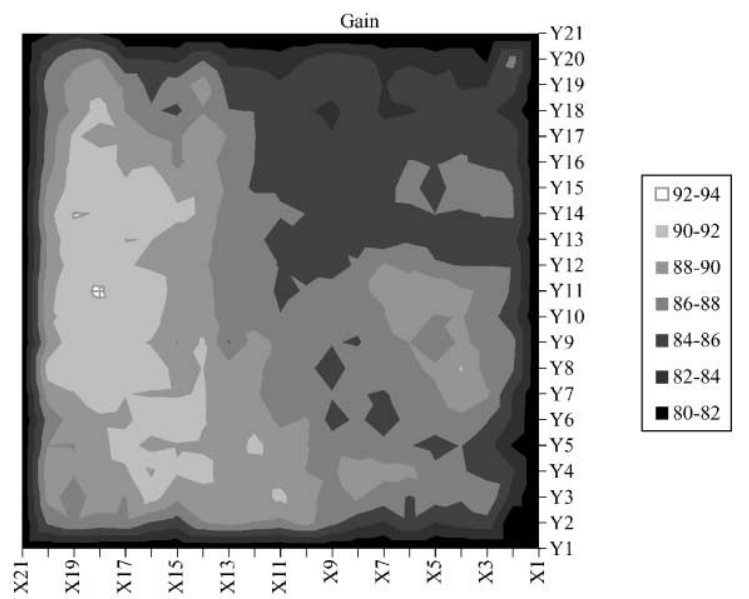

Fig. 7. Surface map of the distribution of the charge gain in a GEM foil. Gain was measured sweeping the detector with an $\mathrm{X}$-ray beam.

Although this technique could be used to check perfectly flat GEMs, it was found that any small deviation from flatness affects the uniformity of reflectivity of the GEM, modulating the CCD scintillation image. It is known that the real gain of the GEMs is mainly dependent on the channel field and tolerant to small angular deviations (ondulation) and we carried on some charge measurements using a scanning system. The GEM was swept with a $30 \mathrm{~mm}^{2}$ X-ray beam with a $5 \mathrm{~mm}$ step. The full system and more detailed results will be presented in a separated paper. Fig. 7 shows the gain map of a $10 \times 10 \mathrm{~cm}^{2}$ GEM 


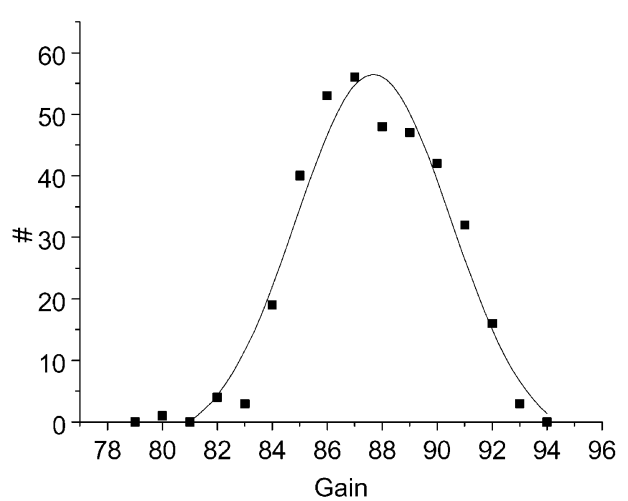

Fig. 8. Distribution of the gain measurements shown in the previous figure. The distribution has a RMS width of $3 \%$.

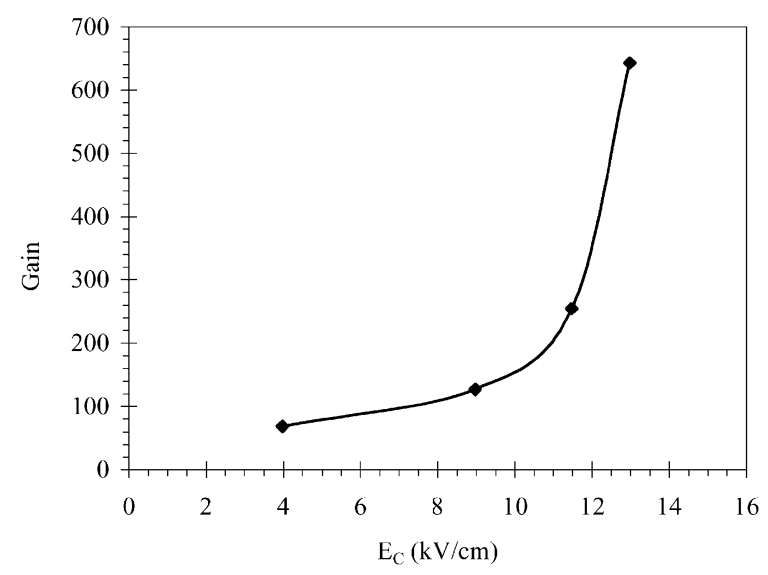

Fig. 9. Measured gain versus collection filed for an $\mathrm{Ar}-5 \% \mathrm{CO}_{2}$ mixture, $\quad G_{\mathrm{EM} 1}=V_{\mathrm{GEM} 2}=300 \mathrm{~V}, \quad E_{\text {Drift }}=1.8 \mathrm{kV} / \mathrm{cm}$, $E_{\text {Tranfer }}=4.5 \mathrm{kV} / \mathrm{cm}$.

and in Fig. 8 is the distribution of gain in the whole surface. The variations of gain on the whole surface was found to be within 5\% RMS. Since this method overcomes the limitations imposed by the reflectivity variations of the GEM, the results are somewhat better. Two other GEM foils, from different manufacturing batches were also analyzed.

\section{The cascaded GEM as a tracking device}

As refered above, in order to improve the sensitivity of the developed imaging system we have used a cascaded double GEM. The amount

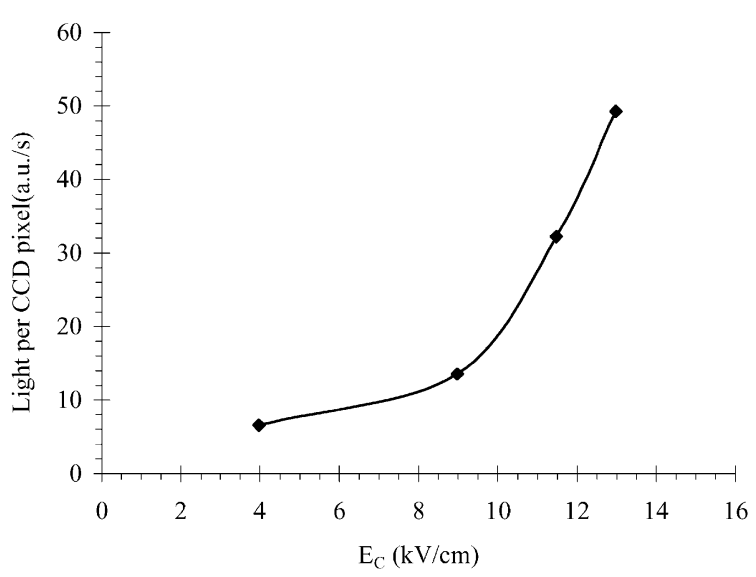

Fig. 10. Ratio of emitted light over secondary electron current versus collection field for an $\mathrm{Ar}-5 \% \mathrm{CO}_{2}$ mixture, $\quad V_{\mathrm{GEM} 1}=V_{\mathrm{GEM} 2}=300 \mathrm{~V}, \quad E_{\text {Drift }}=1.8 \mathrm{kV} / \mathrm{cm}$, $E_{\text {Tranfer }}=4.5 \mathrm{kV} / \mathrm{cm}$.

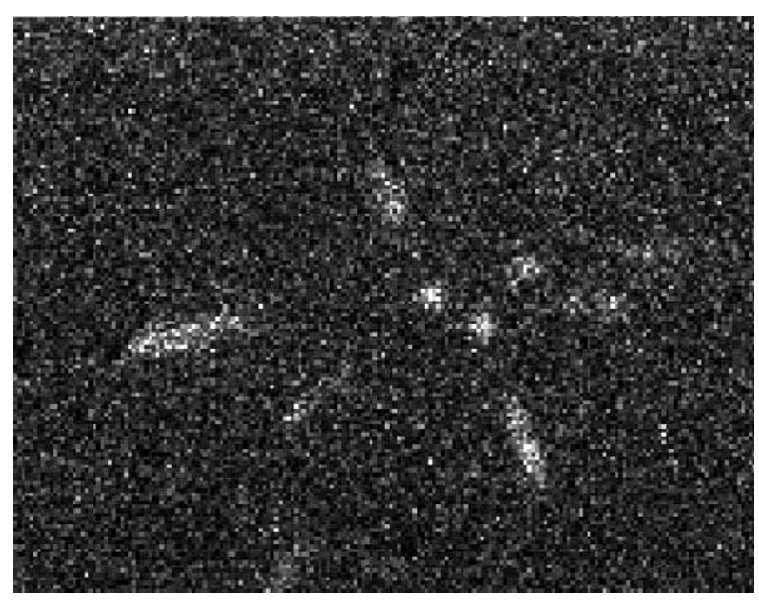

Fig. 11. Scintillation images of partial tracks of alpha particles obtained with an $\mathrm{Ar}-5 \% \mathrm{CO}_{2}$ mixture. $V_{\mathrm{GEM} 1}=$ $V_{\mathrm{GEM} 2}=350 \mathrm{~V}, \quad E_{\mathrm{T}}=5 \mathrm{kV} / \mathrm{cm}, \quad E_{\mathrm{C}}=10 \mathrm{kV} / \mathrm{cm}, \quad \mathrm{CCD}$ binning $=7 \times 7$.

of emitted light can be increased adding a collecting grid placed a few millimeters from the GEM. If the applied field in this additional drift space is high enough, multiplication and light emission occur, with a small degradation of the spatial resolution. The measured variation of gain and light emission versus collection field $\left(E_{\mathrm{C}}\right)$ are shown, respectively, in Figs. 9 and 10 for a collection drift space of $2 \mathrm{~mm}$, resulting in a gain of 7.5 in light emission at $E_{\mathrm{C}}=13 \mathrm{kV} / \mathrm{cm}$. 


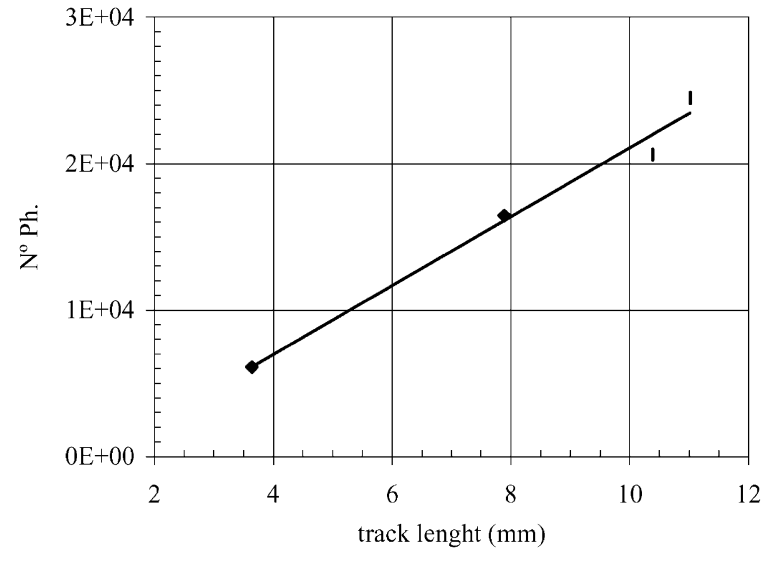

Fig. 12. Number of detected photons versus track length. About 14 photons were detected at the CCD per deposited $\mathrm{keV}$ in the gas.

The global gain obtained with this modification is sufficient to operate our chamber as an imaging device for single events. Fig. 11 shows an image of partial tracks of ${ }^{241} \mathrm{Am}$ alpha particles obtained with a $4 \mathrm{~mm}$ absorption space, corresponding to a mean deposited energy of $150 \mathrm{keV} / \mathrm{mm}$. In order to improve the signal to noise ratio of the CCD we operated it with a pixel binning of $7 \times 7$. The amount of detected light versus track length is plotted in Fig. 12 and from this measured data we can estimate that we detected about 14 photons per deposited $\mathrm{keV}$.

\section{Conclusions}

It has been shown that the optical readout of GEMs is a powerful technique, both for quality control and for imaging. The preliminary results obtained with the double GEM in single event imaging are promising and open a new field of research with GEMs. We are developing a tracking chamber operated in this mode. The optimization of the gaps and the transfer fields in GEMs are of major impoprtance. New gaseous mixtures containing $\mathrm{CF}_{4}$ are also under evaluation and should result in improved performance.

\section{Acknowledgements}

This work was supported by the contract CERN/P/FIS/15199/99 with the Portuguese FCT. Thanks are due to F. Sauli who made available the GEM foils used in this work.

\section{References}

[1] P. Geltenbort, A. Oed, Microstrip anode in noble gases: an active scintillator, Proceedings of the European Workshop on X-Ray Detectors for Synchrotron Radiation Sources, Aussois, France, 30 September-4 October, 1991, p. 107.

[2] F.A.F. Fraga, S.T.G. Fetal, L.M.S. Margato, I. Ivaniouchenkov, R. Ferreira Marques, A.J.P.L. Policarpo, Imaging detectors based on the Gas Electron Multiplier scintillation light, Presented at the Nuclear Science Symposium and Medical Imaging Conference, Seattle, Washington, 24-30 October, 1999, IEEE Trans. Nucl. Sci., accepted for publication.

[3] M.M. Fraga, S.T.G. Fetal, F.A.F. Fraga, E. Antunes, J. Goncalves, C.C. Bueno, R. Ferreira Marques, A.J.P.L. Policarpo, Trans. Nucl. Sci. 47 (3) (2000) 933.

[4] F.A.F. Fraga, S.T.G. Fetal, R. Ferreira Marques, A.J.P.L. Policarpo, Nucl. Instr. and Meth. A 442 (2000) 417.

[5] F. Sauli, Nucl. Instr. and Meth. A 368 (1997) 531. 